TTR

Traduction, terminologie, rédaction

\title{
Du pastiche imaginaire de traduction chez Raymond Queneau : le cas de On est toujours trop bon avec les femmes
}

\section{Tanka Gagné Tremblay}

Volume 26, numéro 1, 1er semestre 2013

Traduction et contact multilingue

Translation and Multilingual

URI : https://id.erudit.org/iderudit/1036954ar

DOI : https://doi.org/10.7202/1036954ar

Aller au sommaire du numéro

\section{Éditeur(s)}

Association canadienne de traductologie

ISSN

0835-8443 (imprimé)

1708-2188 (numérique)

Découvrir la revue

Citer cet article

Gagné Tremblay, T. (2013). Du pastiche imaginaire de traduction chez Raymond Queneau : le cas de On est toujours trop bon avec les femmes. TTR, 26(1), 177-193. https://doi.org/10.7202/1036954ar

\section{Résumé de l'article}

À la frontière des études littéraires et de la traductologie, cet article étudie la dimension pastichielle de traduction dans On est toujours trop bon avec les femmes de Raymond Queneau, prétendument traduit de l'irlandais vers le français. La présente étude cherche à déterminer comment le pseudo-traducteur s'y prend afin d'aveugler et de persuader son lecteur que le texte qu'il tient entre les mains est une véritable traduction. Le postulat repose sur la base du principe qu'il existe une corrélation entre le pastiche littéraire, qui consiste, grosso modo, à imiter à saturation le style d'un auteur singulier, et la pseudo-traduction, qui désigne un texte présenté comme s'il avait été traduit. En effet, il appert que On est toujours trop bon avec les femmes, en tant que " pastiche imaginaire ", répond par essence au critère de saturation du pastiche littéraire tel que l'entend Gérard Genette. Considérant cela, il y a tout lieu de croire que la dimension " pastichielle » de cette pseudo-traduction, laissant présager qu'il y a foncièrement une véritable traduction sous-jacente, se manifeste par la mise en place d'empreintes de traduction, ou d'étrangetés, saturant le texte. La visée de cette étude est donc de révéler ces empreintes dans l'architectonique du roman, en y observant attentivement les divers éléments potentiellement saturés et conditionnels à la crédibilité du canular. 


\title{
Du pastiche imaginaire de traduction chez Raymond Queneau : le cas de On est toujours trop bon avec les femmes
}

\author{
Tanka Gagné Tremblay \\ Université McGill
}

\begin{abstract}
Résumé
À la frontière des études littéraires et de la traductologie, cet article étudie la dimension pastichielle de traduction dans On est toujours trop bon avec les femmes de Raymond Queneau, prétendument traduit de l'irlandais vers le français. La présente étude cherche à déterminer comment le pseudotraducteur s'y prend afin d'aveugler et de persuader son lecteur que le texte qu'il tient entre les mains est une véritable traduction. Le postulat repose sur la base du principe qu'il existe une corrélation entre le pastiche littéraire, qui consiste, grosso modo, à imiter à saturation le style d'un auteur singulier, et la pseudo-traduction, qui désigne un texte présenté comme s'il avait été traduit. En effet, il appert que On est toujours trop bon avec les femmes, en tant que "pastiche imaginaire", répond par essence au critère de saturation du pastiche littéraire tel que l'entend Gérard Genette. Considérant cela, il y a tout lieu de croire que la dimension "pastichielle» de cette pseudotraduction, laissant présager qu'il y a foncièrement une véritable traduction sous-jacente, se manifeste par la mise en place d'empreintes de traduction, ou d'étrangetés, saturant le texte. La visée de cette étude est donc de révéler ces empreintes dans l'architectonique du roman, en y observant attentivement les divers éléments potentiellement saturés et conditionnels à la crédibilité du canular.
\end{abstract}

\begin{abstract}
At the frontier between French studies and translation studies, this article examines the pastichielle dimension of translation in On est toujours trop bon avec les femmes by Raymond Queneau, supposedly translated from Irish into French. This study aims to determine what the pseudo-translator does to persuade the reader that the text is a real translation. This relies on the assumption that a correlation exists between literary pastiche, which essentially consists, in imitating the style of a particular writer to the point of saturation, and pseudo-translation, which presents an original text as a translation. Indeed, it appears that On est toujours trop bon avec les femmes, is an "imaginary pastiche," meeting Gérard Genette's saturation criterion
\end{abstract}


of literary pastiche. With this in mind, there is reason to believe that the pseudo-translation's pastichielle dimension, which fundamentally suggests an actual underlying translation, is expressed through marks of translation, or strangenesses, saturating the text. The aim of this study is to reveal these marks in the architectonic of the novel, by examination of various elements potentially saturated and conditional to the credibility of the hoax.

Mots-clés: pseudo-traduction, pastiche littéraire, R. Queneau, A. Berman, G. Genette

Keywords: pseudotranslation, literary pastiche, R. Queneau, A. Berman, G. Genette

\section{Credo quia absurdum}

(Attribué (faussement) à Saint Augustin)

Entreprendre la lecture de la traduction d'une œuvre littéraire s'effectue d'ordinaire par nécessité. Le cas échéant, le lecteur, n'ayant généralement pas accès au texte source - soit par méconnaissance de la langue, soit par indisponibilité du texte -, accepte le pacte de lecture et, mutatis mutandis, le contrat tacite de traduction qu'il recèle. Dans ces conditions, deux cas de figure peuvent éventuellement se présenter. Dans le premier - le plus fréquent -, le lecteur, sous l'emprise d'un texte au second degré, est à la merci du perfide traducteur: traduttore, traditore. Dans le second - plus pervers -, c'est le statut même de cette traduction qui est mis en cause. Ainsi, le lecteur, assujetti à une pseudo-traduction, c'est-àdire à un texte "which have been presented as translation[s] with no corresponding source text[s] in other languages ever having existed» (Toury, 1995, p. 40), est-il le jouet du prétendu traducteur, qui n'est autre que l'auteur - le scriptor - lui-même.

Dans cette éventualité, au moins deux pistes convergentes envisageables - l'une fonctionnelle, l'autre ludique - semblent éclairer le fallacieux dessein de l'auteur. Pour Gideon Toury, par exemple, grosso modo, vue sous l'optique de l'évolution culturelle et littéraire, la production et la distribution de pseudo-traductions, au même titre que les traductions - «A "translation" will be taken to be any target-language utterance which is presented or regarded as such within the target culture» (Toury, 1985, p. 20) -, 
permettent d'introduire des nouveautés au sein d'une culture conservatrice et récalcitrante au changement. Ainsi, posant le fait que «translations which deviate from sanctioned patterns [...] are often tolerated by a culture to a much higher extent than equally deviant original compositions» (Toury, 2005, n.p.), Toury affirme qu' in fine, "by presenting a text as if it were translated ${ }^{1}$, thus lowering the threshold of resistance to the novelties it may hold in store and enhancing their acceptability, along with that of the text incorporating them as a whole» (Toury, 2005, n.p.). En outre, en l'espèce, pour plusieurs observateurs (notamment Yahalom, 1980, 1981 et Herman, 1990, 2003), il semble que cette thèse s'applique apparemment parfaitement au système littéraire français du XVIII ${ }^{\mathrm{e}}$ siècle.

Dès le siècle suivant, cependant, de Prosper Mérimée à Umberto Eco, en passant par Pierre Louÿs et Boris Vian, il en retourne tout autrement avec les manifestations, de plus en plus fréquentes, de pseudo-traducteurs folâtres. Ce qui ne les empêche pas d'agir consciencieusement. Conséquemment, même si pour certains, il ne s'agit que de "faire une supercherie" comme on fait un roman ou un poème, c'est-à-dire sans y croire ni demander qu'on y croie» (Avni, 1985, p. 30), d'aucuns s'y appliquent soigneusement, au-delà du «simple goût fabulateur» (Eco, 1985, p. 13), avec l'envie réelle de mystifier l'affable lecteur ${ }^{2}$. Ainsi s'érige, justement, l'entreprise de Raymond Queneau lorsqu'il rédige en 1947 sous le nom de Sally Mara, en marge de son œuvre plus officielle, son roman On est toujours trop bon avec les femmes ${ }^{3}$ (Mara, 1947), qui relate les tribulations d'une bande d'insurgés irlandais ayant pris d'assaut la poste centrale de Dublin le lundi de Pâques du 24 avril 1916. Rappelons que le roman, traduction supposée de l'irlandais vers le français, paraît précisément le 8 novembre 1947, soit un an jour pour jour, suivant les achevés d'imprimer, après

1. En gras dans le texte.

2. Sur la question de la pseudo-traduction et de tout ce qu'implique cette «mise en scène» particulière de «l'altérité», v. Collombat (2003).

3. Dorénavant désigné dans ce texte par l'acronyme OETTBALF. Précisons que Queneau ne réitérera pas l'exercice de style, se contentant d'user de l'artifice hétéronymique une autre fois au moins le temps de publier le Journal intime de Sally Mara en 1950 chez le même éditeur. Les Euvres complètes de Sally Mara paraîtront chez Gallimard en 1962 sous le nom de Raymond Queneau. 
le roman noir américain J'irai cracher sur vos tombes de Vernon Sullivan, prétendument traduit de l'américain par Boris Vian (Sullivan, 1946).

Notre hypothèse est la suivante: OETTBALF, en tant que "pastiche imaginaire» - "texte endossé par l'hétéronyme» dans lequel «l'auteur $[. .$.$] forge de toutes pièces un idiolecte jusqu'alors$ inconnu, qui ne provient d'aucun texte préexistant» (Genette, 1992, p. 175) -, répond essentiellement au critère de saturation du pastiche littéraire tel que l'entend Gérard Genette dans Palimpsestes. Ainsi, estimant comme Genette que «l'essence même du pastiche implique une saturation stylistique» (Genette, 1992, p. 221), c'est-à-dire l'utilisation excessive d'un trait stylistique caractéristique d'un auteur, il y a tout lieu de croire que la dimension «pastichielle» d'une pseudo-traduction telle qu'OETTBALF laissant présager qu'il y a foncièrement une véritable traduction sous-jacente - se manifeste par la mise en place d'empreintes de traduction, ou d'étrangetés, saturant le texte et le paratexte. La visée attendue repose donc sur la mise en relief de ces empreintes dans l'architectonique du roman et tend à générer, voire à régénérer son modèle de compétence et sa matrice d'imitation. Comme le note Genette dans Palimpsestes:

Imiter [...] suppose [...] la constitution préalable [...] d'un modèle de compétence dont chaque acte d'imitation sera une performance singulière. [...] Entre le corpus imité [...] et le texte imitatif, s'interpose inévitablement cette matrice d'imitation qu'est le modèle de compétence ou, si l'on préfère, l'idiolecte du corpus imité. [...] [Bref,] imiter précisément, dans son éventuelle singularité, un texte singulier, c'est d'abord constituer l'idiolecte de ce texte, c'est-à-dire identifier ses traits stylistiques et thématiques propres, et les généraliser, c'est-à-dire les constituer en matrice d'imitation, ou réseau de mimétismes, pouvant servir indéfiniment. (Genette, 1992, p. 108-109)

Suivant ces propos, tout porte à croire que Queneau, avant d'entreprendre l'écriture d'OETTBALF, a d'abord élaboré une matrice, constituée d'un certain nombre de marques de traduction, pour asseoir solidement son récit. Comme si l'auteur avait préalablement établi une liste de formules et de calques à imiter. Aussi projetons-nous, à chaque fois qu'il en sera possible 
et au moment opportun, de collationner l'édition princeps, de 1947, d'OETTBALF, aux notes de composition du texte, afin de retrouver des traces de cette hypothétique matrice.

Pour ce faire, nous proposons d'emboîter le pas à Antoine Berman et de mettre en application les idées qu'il développe dans ses ouvrages sur la traduction, La traduction et la lettre ou l'auberge du lointain (Berman, 1999), Pour une critique des traductions, John Donne (Berman, 1995) et L'épreuve de l'étranger (Berman, 1984), afin de relever les marques ou étrangetés traductives saturant le roman et ainsi mettre à jour sa dimension pastichielle de traduction. Plus précisément, notre démarche prend virtuellement la forme d'une critique de traduction, au sens bermanien du terme - «analyse rigoureuse d'une traduction, [...] dégagement de la vérité d'une traduction» (Berman, 1995, p. 13-14; en italique dans l'original) -, en considérant $O E T T B A L F$, étant donné son abondance en étrangetés, à l'égal d'une «traduction-de-la-lettre». Pour Berman, qui préconise ce type de traduction, «amender une œuvre de ses étrangetés pour faciliter sa lecture n'aboutit qu'à la défigurer et, donc, à tromper le lecteur que l'on prétend servir» (Berman, 1999, p. 73). Conséquemment, «ouvrir au niveau de l'écrit un certain rapport à l'Autre, féconder le Propre par la médiation de l'Étranger»(Berman, 1984, p. 16) [telle est la visée de la traduction] suppose donc la mise en valeur et l'incorporation au sein de la traduction d'un certain nombre d'étrangetés. Ainsi suit-elle, à rebours, ce que Berman appelle l'analytique de la traduction, c'est-à-dire l'analyse et la mise à jour du système de déformation des textes, à savoir, "[des] tendances et [des] forces qui dévient la traduction de sa pure visée»(Berman, 1999, p. 49).

\section{De Berman à Queneau}

Le recours à l'approche bermanienne de la traduction pour mettre en analyse le texte de Queneau peut sembler douteux et susciter bon nombre de questions, à savoir, a priori, s'il est pertinent de faire appel à ce type de méthode analytique dans le cadre de l'étude d'une pseudo-traduction, l'exercice péchant par défaut d'applicabilité. Nous faisons le pari qu'OETTBALF, attendu la pléthore d'étrangetés s'y trouvant, peut raisonnablement et partiellement être considéré au même titre qu'une traductionde-la-lettre. Conséquemment, le recours - du moins en partie et 
sous certaines réserves que nous signalerons le cas échéant - à la théorie bermanienne nous apparaît la meilleure voie possible afin de relever les marques ou étrangetés langagières de traduction saturant le texte du roman, parmi lesquels abondent, comme nous le constaterons, graphies phonétiques et emprunts linguistiques.

\section{De l'oral au scriptural : aspects phonétiques et graphiques}

Débordant le cadre de la simple transcription, la transposition de l'oral au scriptural, à graphie phono-stylistique, est omniprésente dans le texte. Apparaissent ainsi, dans OETTBALF, de multiples faits de déformation et de réformation syntaxique, allant du plus simple amalgame syntaxique à ellipse vocalique : d'la [de la] (p. 63) ptêt'même [peut-être même] (p. 140), à la plus remarquable agglutination: mormoualeneuf [mord moi le nœud] (p.62), auxquels s'ajoutent calembours: décore-homme [décorum] (p.12), mots-valises: barricadure [amalgame phonétique de barricade et d'armature] (p. 56), pseudo-blésité: s'exclaffer [s'esclaffer] (p. 159), transcription phonétique mono et trigrammatique: koua [ $k$ pour $q u$ et oua pour oi = quoi] (p. 63), kant-à-soi [k pour qu = quant-àsoi] (p. 64), ou pire encore: sortè-je [è pour $a i=$ sortai-je, prétérit erroné du verbe sortir: sortis-je] (p. 17) et onomatopée: en outre la troublante figure onomatopéique $B z z z$ (p. 175), faisant écho à la bande dessinée, apparaissant en fin de texte, simulant, de prime abord, le sifflement provoqué par le passage d'un projectile.

Si les métagraphes ${ }^{5}$ évoqués précédemment sont certes mimétiques de l'oral, donnant l'illusion de sa simultanéité et de sa rythmique, ils s'avèrent surtout sigillés d'un sceau empreint de ludisme. À cet égard, l'exemple de l'onomatopée Bzzz est probant: occupant à elle seule une pleine page, un chapitre entier y étant consacré, mais surtout, donnant la réplique à l'ordre de tir du commodore Cartwright, qui justement fait «parler» les armes: «-Feu! / C'était le commodore Cartwritht qui avait tenu à diriger en personne le définitif bombardement / - Bzzz, fait l'obus» (p. 174-175). Comme le note Daniel Delbreil: «Lobjet qui fait du bruit, l'objet émetteur donc, serait à l'origine de la véritable

4. Sauf indication contraire, nous renvoyons à Mara (1947).

5. V. Groupe $\mu$ (1982), p. 52 et p. 65 : «Opération modifiant l'aspect écrit du mot sans attenter à sa forme phonétique.» 
onomatopée»(Delbreil, 1993, p.137). Plus encore: «Tous ces bruits et cris [onomatopéiques], quelle que soit leur origine exacte, peuvent être considérés comme des "bruits naturels", des bruits qui permettent à la chose de parler et même de "beugler"» (Delbreil, 1993, p. 138). Ainsi, acquérant de la sorte la valeur du «personnage» participant de l'action, l’obus, désacralisé et détourné de son statut ontologique premier, frappe l'imaginaire et par le truchement de son étrangeté métaphysique, apparaît comme le deus ex machina de l'histoire.

Mais si l'intention métagraphique est ludique, elle n'est pas nécessairement gratuite. On observe ce type de situation dès la première phrase d'OETTBALF, lorsque surgit l'étrange lexème kasseul, souligné non seulement par les guillemets, mais également par le discours métalinguistique qui l'accompagne:

- Dieu sauve le Roi! s'écria l'huissier qui avait été valet de lord dans le Sussex, pendant trente-six ans, puis son maitre disparut dans le naufrage du Titanic sans laisser d'héritier, ni de sterling, pour entretenir le «kasseul», comme on dit de l'autre côté du canal Saint-George. (p. 9)

Par delà la simple caricature anglicisante, la transcription phonétique de castle accuse sarcastiquement et semble railler les caractéristiques prosodiques du locuteur français se hasardant à l'anglais. Comme si l'auteur, au risque de se démystifier, cherchait à rapprocher le lexème du système phonétique de la langue française, déconstruisant, à l'encontre de la mouvance littéraliste bermanienne, la traduction. Ailleurs, on remarque un procédé à graphie phono-stylistique semblable lors de la francisation de certains mots d'Outre-Manche originellement admis dans le lexique français: smokinge [smoking] (p. 12), ouisqui [whisky] (p. 28 sq.), angliche [english] (p.62 et 93), vatère-prouf (p.10), ouatères (p. 62), vécés (p. 93) [variations sur Waters et W.C. :], ou étranger à celui-ci: lavatories [lavatory] (p. 16 et beaucoup d'autres), britiche [british] (p. 51-52), englishes [english] (p. 82), misteur [mister] (p. 154), darligne [darling] (p. 187-188). A croire que le locuteur, en la personne du traducteur, ignorant leur prononciation originale, les a adaptés à sa propre langue, effaçant, ipso facto, toute trace de traduction. 
Or, si ces métagraphes séloignent sensiblement du type de traduction prôné par Berman, demeure pourtant, au-delà de toute approche traductive, cette impression d'étrangeté ou de défamiliarisation. Mais est-ce bien suffisant pour piéger et duper le lecteur, qui risque d'y percevoir une badinerie, voire de penser que ce n'est pas très sérieux et crédible pour une traduction? Certes, Queneau, amoureux de l'orthographe phonétique, mais inconnu du grand public à l'époque, n'est pas reconnaissable. Mais il ne faudrait tout de même pas pousser le jeu trop loin, au risque d'éveiller les soupçons et de s'enferrer! En fait, cette impression, lorsquétendue à l'ensemble des procédés évoqués, comme nous allons l'observer, ne doit l'essentiel de son impact qu'à sa collocation avec un autre artifice bien sournois, entraperçu précédemment, consistant à semer le texte d'idiotismes et d'emprunts linguistiques judicieusement choisis et repérables par le lecteur moyen, lui laissant entrevoir les sources originelles et sous-jacentes au texte tenu entre ses mains.

\section{L'emprunt linguistique : entre pérégrinisme et xénisme}

De par son aspect apparemment authentique et inaltéré, force est de constater que l'emprunt linguistique s'avère, dans le cas qui nous occupe, le procédé langagier le plus efficace et le plus persuasif. Imprégnant ponctuellement le texte de ces «mots sentis comme étrangers» (Deroy, 1956, p. 224) que sont les pérégrinismes (Dupriez, 1984) ${ }^{6}$ et les xénismes (Dumont, 1983) ${ }^{7}$, le scriptor y va désormais et tout de bon de l'ultime et décisive tentative mystificatrice.

Une fois de plus, la manœuvre apparaît très tôt dans le texte, dès les premiers mots, à vrai dire, au contact du calque de la formule hymnique britannique bien connue, "God Save the King", devenu «Dieu sauve le roi» (p. 9, 12, 22, 29, 79). Il faut dire que le

6. Cité par Ly (1999, p. 90): «Éléments linguistiques empruntés à une langue étrangère, au point de vue des sonorités, graphiques, mélodies de phrase aussi bien que des formes grammaticales, lexicales ou syntaxiques, voire même des significations ou des connotations.»

7. Cité par Ly (1999, p. 90): «Terme étranger qui désigne une réalité inconnue ou très particulière et dont l'emploi s'accompagne, nécessairement, d'une marque métalinguistique qui peut être soit une paraphrase descriptive, soit une note explicative en bas de page quand il s'agit d'un texte.» 
pérégrinisme est bien trouvé et représentatif, puisque les Irlandais, républicains et catholiques, à la différence des Britanniques, en veulent justement à la couronne du roi d'alors, George V, et à la Parole de leur Dieu, allant jusqu'à le jurer! Les blasphèmes «nom de Dieu» (p.110) et «sacré nom de Dieu» (p.159) répondent d'ailleurs à ce «Dieu sauve le roi».

Nulle surprise, aussi, de voir sourdre du texte toute une panoplie d'éléments lexicaux ou syntactiques issus directement de la langue anglaise, allant du simple morphème: yes (p. 95), sir (p. 26, 59, 84, 90 et 91), au syntagme: "what do you say» (p. 29), entre lesquels abondent lexèmes: mister (p. 10), lobster (p. 69), ladies (p. 148 et p. 160), et même superladies (p. 160), et locutions: Sackville Street et Eden Quay (p. 9, 23, 46, 111 et 118), O'Connell Bridge (p. 14, 40, 41, 77, 113 et 130), Finnegans wake (p. 13, 15, 24, 29, 57, 61, 112, 150 et 185), Civil Service (p. 19), Post Office (p. 39, 40, 43 et 94), By Jove (p. 112). Le texte est marqué, balisé, comme si on avait voulu solliciter et entraîner le lecteur à suivre, peut-être inconsciemment, une piste piégée. Ces marques de traduction, ou disons plutôt, d'absence de traduction (!), repérables dans le texte, sont, proportionnellement, d'autant plus crédibles qu'elles sont nombreuses. Comme si l'épreuve de l'étranger était synonyme de traduction. Comme si on avait voulu demeurer le plus près possible de la lettre du texte original pour en conserver la transparence et l'authenticité, pour en rendre l'étrangeté. Comme si, butant sur des étrangetés, le lecteur avait l'impression d'être en contact avec l'étranger. Et Queneau de déclarer, dans les versions préliminaires de la préface d'OETTBALF: «Quant à la traduction, elle a tous les mérites d'une bonne traduction; on dirait à peine que c'en est une $^{8}$.» Si Queneau, sous les traits de l'éditeur - ce qui n'est plus le cas dans la préface qui sera publiée -, met l'accent sur l'authenticité et la fidélité de la «traduction", c'est à peine s'il ne dévoile pas sa mystification, à trop insister sur ces détails. Mais l'extrait n'est pas retenu et n'apparaîtra jamais dans le texte.

Par ailleurs, à observer attentivement les notes de composition du texte, nulle surprise d'y découvrir également un feuillet

8. Les archives d'OETTBALF conservées au Centre de Documentation Raymond Queneau à Verviers en Belgique font notamment état d'une préface manuscrite et d'une épreuve de cette préface dans lesquelles on retrouve, avec de très légères variations de l'une à l'autre, ces propos (v. «Notes et fragments », classeur 60, partie $\mathrm{A}$, section 2). 
(reproduit à la page suivante) ${ }^{9}$ répertoriant une série de termes français et leur traduction en anglais, à tendance érotique. S'ils n'apparaissent pas, eux non plus, dans le texte, du moins laissentils croire qu'il y a préméditation.

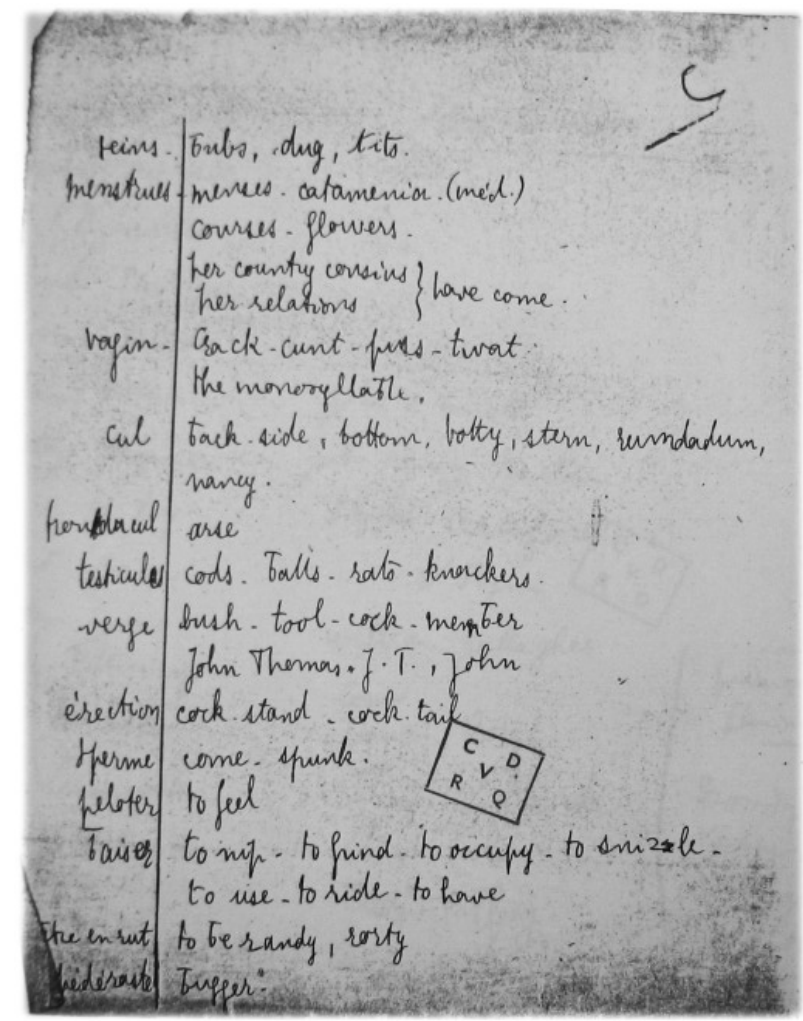

Et si leur présence est fortuite et inconséquente - ce dont nous doutons, connaissant la méticulosité de Queneau -, au moins laissent-ils présager l'existence d'une matrice d'imitation, c'està-dire de marques de traduction préalablement déterminées et ensuite greffées au texte. Par ailleurs, nous remarquons, du point de vue onomastique, que les noms des protagonistes,

9. Tiré des archives d'OETTBALF conservées au Centre de documentation Raymond Queneau (v. «Notes et fragments», classeur 60, partie A, section 1), 
qu'ils soient français (Théodore Durand), anglais (Gertie Girdle; le commodore Sidney Cartwright, Teddy Mountcatten) ou Irlandais (Corny Kelleher, John Mac Cormack, Gallager, Mat Dillon, Cisey Caffrey, Chris Callinan, Larry O'Rourke, Teddy Lanark et Shan Dromgour) apparaissent bien, eux également, à l'égard de leur origine respective, véridiques et authentiques: indice supplémentaire de la proximité de la «traduction» avec le texte original.

Mais l'emprunt ne devient réellement systématique et significatif qu'avec l'usage massif d'idiotismes et d'expressions idiomatiques reprenant, en français ou par hybridation, la composition morphosyntaxique anglaise: Dieu gracieux (p. 32) ou God gracieux (p. 16, 17 et 22) [variations sur gracious God et good gracious], ob Dieu, ob mon roi [oh God, oh my lord] (p. 37), Navy royale [Royal Navy] (p.95), self-contrôle [self-control] (p. 96), le courage de la force [courage of the strength] (p. 111), état prégnant [pregnant] (p.130), bovine obstination [bovine obstinacy] (p.143), définitif bombardement [definitive bombardment] (p. 174), ou s'inscrivant simplement comme tel, en anglais: correct (p. 11 sq.), at home (p. 12), corned-beef (p.123, 124 et 126), lunch (p. 125, 126 et 146), docks (p. 127), yards (p. 130), miss (p. 154). De nouveau, le texte n'est qu'un attrape-nigaud visant à faire croire au lecteur qu'il a sous les yeux une véritable traduction et que les emprunts linguistiques sont des restes, des vestiges du texte original étranger, mi-traduits ou non traduits, par omission ou par négligence. Comme si on voulait que le lecteur sente et perçoive l'imprégnation de l'anglais dans un texte dont la traduction reste inachevée, et donc, d'autant plus perceptible.

Quel rapport avec l'irlandais? Car n'oublions pas, après tout, qu'OETTBALF est, a priori, une traduction de l'irlandais! Eh bien, à Dublin, à l'époque, c'est bien en anglais que l'on parle, du moins le plus souvent, et non en irlandais. Parions aussi que l'insertion abusive de mots irlandais n'aurait eu pour effet que d'alourdir et de surcharger le texte, risquant le décrochage du lecteur. Ainsi, comme marque d'étrangeté ou d'extranéité, l'insertion de l'anglais est d'autant plus réaliste et intelligible que suffisante. Ce qu'observe également Philip Morey: 
The dialogue of characters who are native speakers of English, which includes nearly everyone in the two Irish novels, is almost invariably reported in French. [...] The sparing insertion of English words or calques in the dialogue or reported speech attempts to strike a balance between presentational realism and intelligibility. In the case of English speakers it reminds the reader of the foreign location. (Morey, 1981, p. 830-831)

Mais l'insertion parcimonieuse, pour reprendre les mots de Morey, de mots étrangers de langues autres que l'anglais est également garante d'étrangeté! Aussi, rien d'étonnant à observer pareille situation pour d'autres langues d'emprunt comme l'irlandais: Eire [Irlande] (p. 15 et 114), l'allemand: anschauung (p. 47), le celte: ankou (p. 47, 53 et 54), le français: d'un seul coup d'un seul (p. 47) [à en croire les notes infrapaginales du traducteur en bas de page, anschauung, ankou et d'un seul coup d'un seul sont des variations sur intuition] et le latin: speculatrice (p. 93) ["de speculatrix, expionne", note à nouveau le traducteur]. Cela dit, il convient de distinguer les trois locutions précédentes, de l'ordre du xénisme, en ce qu'elles apparaissent, précisément, en italiques et accompagnées d'une note explicative en bas de page.

Phénomène plutôt inusité hors des études critiques, l'usage de la note dans les textes de fiction, à en croire les propos de Shari Benstock, s'avère assez particulier:

Footnotes in fictional texts do not necessarily follow the rules that govern annotation in critical texts: they may or may not provide citation, explication, elaboration, or definition for an aspect of the text; they may or may not follow "standard form"; they may or may not be subordinate to the text to which they are affixed. Most significant, they belong to a fictional universe, stem from a creative act rather than a critical one, and direct themselves toward the fiction and never toward an external construct, even when they cite "real" works in the world outside the particular fiction. (Benstock, 1983, p. 204205)

Autant dire que l'annotation en fiction, libre et subversive, crée de curieuses situations. Or, dans OETTBALF, à une exception près (p. 71), la note apparaît sous la plume du prétendu traducteur. Force est de constater que la note est alors bel et bien de l'ordre de l'appareil critique et que c'est avec cet appareil que Queneau joue. 
Mais ne précipitons pas les choses, l'affaire est délicate! Comme l'observe Jacqueline Henry, la note du traducteur:

est un paratexte allographe, c'est-à-dire écrit par un tiers qui n'est donc ni l'auteur ni un sujet/personnage du livre [...]. Elle n'apparaît que dans des textes traduits, c'est-à-dire écrits dans une langue autre que celle de l'original. Et elle est donc le fait de ce tiers dont la tâche est de restituer l'œuvre première dans un contexte linguistique, culturel, géographique, voire temporel, second. (Henry, 2000, p. 230)

Qui plus est, c'est à ce tiers, le traducteur, qu'appartient la tâche de confronter le lecteur à l'épreuve de l'étranger, ou comme le note à nouveau Henry:

C'est à lui qu'il incombe de rendre possible la rencontre de l'Autre, et donc de choisir tantôt d'expliciter, par différents moyens, dont la N.d.T, tantôt de laisser au texte proposé à son lecteur une part d'ombre et d'étrange, tantôt de gommer l'altérité, en «naturalisant» ou «acclimatant» l'œuvre. (Henry, 2000, p. 237)

On l'aura compris, manifestement, à en croire les propos susdits, le soi-disant traducteur/commentateur d'OETTBALF, bien malin, opte pour les deux premières voies, poussant ainsi l'étrangeté, mais également le jeu, à son comble. À la lecture de ses notes, c'est à croire que le scriptor, qui tient, bien entendu, à éviter, sous le masque du traducteur, toute équivoque, se faisant même pour l'occasion pseudo-étymologiste - brayette [et non braguette], "pièce du costume masculin fort commune en Irlande» (p. 90) -, se joue éperdument du lecteur! Aussi, c'est à une véritable parodie de la note explicative que nous assistons, alors que le traducteur, tantôt désinvolte: "Après l'avoir chantée [le God Save the King], on se sépare» (p. 60), "Intraduisible en français [le terme latin speculatrix], langue un peu pauvre comme chacun sait» (p.93), tantôt confus: ankou, "celticisme pour "intuition"», anschauung, "germanisme pour "ankou"», d'un seul coup d'un seul, "gallicisme pour "anschauung”» (p.47), obscurcit le texte plus qu'il ne le clarifie.

On ne s'étonnera pas non plus de ce que la seule note de l'auteur d'OETTBALF soit tout aussi insolite et troublante: «il 
y a là un léger anachronisme ["on voit qu'on est dans le pays de James Joyce”, observe Caffrey], mais Caffrey, étant analphabète, ne pouvait savoir en 1916 qu'Ulysses n'avait pas encore été publié» (p. 71), alors qu'un illettré évoque un monde romanesque auquel, de son état, il n'a forcément pas accès. Mais il ne faudrait tout de même pas forcer la note et laisser entrevoir le ludisme qui se cache sous ces propos! Après tout, le lecteur n'est peut-être pas si sot; à tant faire, il pourrait bien finir par se douter de quelque chose.

En tout état de cause, il n'y a pas à dire, sous le masque du traducteur Michel Presle et de l'auteur Sally Mara se dissimule un génie créateur étrangement minutieux, voire maniaque! Si le scriptor s'amuse, aux dépens du lecteur, laissant parfois entrapercevoir sa griffe et autres marques indicielles, au risque de dévoiler sa véritable identité, il est également, paradoxalement, attentif au moindre détail et veille au grain, piégeant au détour d'une phrase quelque peu singulière et hagarde d'aucuns parmi les lecteurs pourtant bien entraînés. À quoi bon tous ces efforts alors? Gageons qu'au fond, tout ce que désire Queneau, c'est que le lecteur, même le plus incrédule, ait sa dose de plaisir!

\section{Du pari quenien ou de la réception d'OETTBALF}

Cuvre atypique et singulière, OETTBALF s'inscrit en marge $\mathrm{du}$ corpus romanesque quenien, avec le Journal Intime, en ce que l'auteur, ou plutôt le scriptor, Queneau, y adopte un ton beaucoup plus salace et noir que dans ses autres romans. OETTBALF partage cependant au moins un trait avec ses semblables en celui de la minutie du détail, qui révèle une rigueur toute classique de l'auteur, indispensable à la bonne réussite de l'opération canularesque.

Car OETTBALF est bien une mystification, qui plus est, de l'ordre de la pseudo-traduction, dont la visée est de persuader un Lecteur Modèle que le texte qu'il a sous les yeux est une véritable traduction. Comment Queneau s'y prend-il? Telle est la question que la présente étude s'est efforcée d'examiner.

Récapitulons. Notre hypothèse de départ est la suivante: $O E T T B A L F$, en tant que pastiche imaginaire de traduction laissant présager à tort l'existence d'un autre texte correspondant dans une autre langue, l'original -, répond au critère de saturation du pastiche littéraire tel que l'entend Gérard Genette, en ce qu'il regorge d'empreintes de traduction, ou d'étrangetés, saturant 
le texte et le paratexte. Notre intention est donc de relever ces empreintes et de générer, voire de régénérer le modèle de compétence et la matrice d'imitation du roman. Le résultat n'est malheureusement guère probant. Certes, étrangetés langagières de traduction, graphies phonétiques et emprunts linguistiques confrontent de plain-pied le lecteur à l'épreuve de l'étranger. L'ennui, en tout état de cause, est que Queneau semble parfois se relâcher, fléchir devant son entreprise fallacieuse. Comme s'il ne pouvait plus se retenir de pouffer, comme s'il allait éclater de rire. Le discours métalinguistique entourant le lexème kasseul est, à cet égard, assez révélateur! En résulte une tension dramatique parfois facétieuse qui laisse forcément le lecteur perplexe. Ainsi, il est en droit de se demander jusqu'à quel point Queneau tenait à faire perdurer l'imposture, tenait, simplement, à mystifier le lecteur.

Rappelons que la duperie, plutôt fugace, n'aura duré, tout au plus, que deux mois. Qui sait ce que Queneau avait réellement derrière la tête! Ce qui nous amène également à douter de l'existence d'un modèle de compétence sous-jacent au roman, en ce que les étrangetés que nous avons repérées semblent plutôt avoir été semées, çà et là, en cours d'écriture. S'il appert tout de même de ce repérage d'étrangetés une sorte de modèle de compétence pouvant vraisemblablement être repris indéfiniment et nous poussant à croire au potentiel d'analyse du phénomène des pseudo-traductions à partir de notre méthode ${ }^{10}$, il subsistera toujours une faille apparente dans ce type d'exercice en ce que l'auteur - le véritable! - semble toujours tenté, à un moment ou l'autre de sa vie, de crier haut et fort: «acta est fabula, c'est moi qui l'ai fait!», comme Queneau, quinze ans après le canular original, lors de la publication des CEuvres complètes de Sally Mara. En espérant que l'auteur -l'Autre -, même imaginaire, ne s'en mêle pas!

\section{Références}

AVNI, Ora (1985). «Mystification de la lecture chez Mérimée. Lira bien qui rira le dernier». Littérature, 58, p. 29-41.

10. Il faudrait refaire l'exercice notamment avec J'irai cracher sur vos tombes de Vernon Sullivan, alias Boris Vian. 
BENSTOCK, Shari (1983). «At the Margin of Discourse: Footnotes in the Fictional Text». Publications of the Modern Language Association of America, 98, 2, p. 204-225.

BERMAN, Antoine (1984). L'épreuve de l'étranger. Paris, Gallimard.

BERMAN, Antoine (1995). Pour une critique des traductions: John Donne. Paris, Gallimard.

BERMAN, Antoine (1999). La traduction et la lettre ou l'auberge du lointain. Paris, Seuil.

COLLOMBAT, Isabelle (2003). «Pseudo-traduction: la mise en scène de l'altérité». Le Langage et l'Homme, XXXVIII, 1, p. 145-156.

DELBREIL, Daniel (1993). «Quenonomatopées». Temps Mêlés. Documents Queneau, $150+57-60$, p. 135-156.

DEROY, Louis (1956). L'emprunt linguistique. Paris, Les Belles Lettres.

DUMONT, Pierre (1983). Le français et les langues africaines au Sénégal. Paris, Karthala.

DUPRIEZ, Bernard (1984). Gradus. Les procédés littéraires. Paris, Union générale d'Éditions.

ECO, Umberto (1985). Le nom de la rose. Paris, Grasset.

GENETTE, Gérard (1992 [1982]). Palimpsestes, la littérature au second degré. Paris, Seuil, coll. «Points Essais».

GROUPE $\mu$ (1982). Rhétorique générale. Paris, Seuil.

HENRY, Jacqueline (2000). "De l'érudition à l'échec: la note du traducteur». Meta, XLV, 2, p. 228-240.

HERMAN, Jan (1990). «Le Procès Prévost traducteur. Traduction et pseudo-traduction au dix-huitième siècle en France». Arcadia. Zeitschrift für vergleichende Literaturwissenschaft, 25, 1, p. 1-9.

HERMAN, Jan (2003). «La pseudo-traduction de romans anglais dans la première moitié du XVIII ${ }^{e}$ siècle. Analyse titrologique». In A. Cointre, A. Lautel et A. Rivara, dir. La Traduction romanesque au XVIII siècle. Arras, Artois Presses Université, p. 11-25

LY, Amadou (1999). «Le pérégrinisme comme stratégie textuelle d'appropriation de la langue d'écriture». In Lise Gauvin, dir. Les langues du roman. Du plurilinguisme comme stratégie textuelle. Montréal, Les Presses de l'Université de Montréal, p. 87-100.

MARA, Sally [pseudonyme de Raymond Queneau] (1947). On est toujours trop bon avec les femmes. Paris, Les Éditions du Scorpion.

MARA, Sally [pseudonyme de Raymond Queneau] (1950). Journal intime. Paris, Les Éditions du Scorpion.

MOREY, Philip (1981). "English Words in Queneau». The Modern Language Review, 76, 4, p. 823-838. 
QUENEAU, Raymond (1962). Les Euvres complètes de Sally Mara. Paris, Gallimard, coll. «L'imaginaire».

SULLIVAN, Vernon [pseudonyme de Boris Vian] (1946). J'irai cracher sur vos tombes. Paris, Les Éditions du Scorpion.

TOURY, Gideon (1985). «A Rationale for Descriptive Translation Studies ». In Theo Hermans, dir. The Manipulation of Literature. New York, St. Martin's Press, p. 16-41.

TOURY, Gideon (1995). "Pseudotranslations and their Significance», Descriptive Translation Studies and Beyond. Amsterdam/Philadelphia, John Benjamins, p. 40-52.

TOURY, Gideon (2005). «Enhancing Cultural Changes by Means of Fictitious Translations». In Eva Hung, dir. Translation and Cultural Change: Studies in History, Norms and Image-Projection. Amsterdam/Philadelphia, John Benjamins, non pag. [http://www. tau.ac.il/ toury/works/fict.htm].

YAHALOM, Shelly (1980). «Du non-littéraire au littéraire: sur l'élaboration d'un modèle littéraire au XVIII siècle». Poétique, 44, p. 406-421.

YAHALOM, Shelly (1981). «Le système littéraire en état de crise». Poetics Today. 2, 4, p. 143-160.

Tanka Gagné Tremblay

2320, rue L.-O.-David Montréal (Québec) H2E 1M1

CANADA tanka.tremblay@gmail.com 